\title{
Graft-Associated Elizabethkingia meningoseptica Bacteremia Managed With Long-Term Oral Ciprofloxacin and Rifampicin
}

\author{
Margaret Omatsone ${ }^{\mathrm{a}, \mathrm{b}}$, Harold Katner ${ }^{\mathrm{a}}$, Colleen Bradley ${ }^{\mathrm{a}}$, \\ Anthonia Omatsone ${ }^{\mathrm{a}}$
}

\begin{abstract}
Elizabethkingia meningoseptica is an uncommon pathogen that has been associated with nosocomial infections and can pose a therapeutic challenge. It is resistant to most conventional agents used in treatment of gram-negative organisms and as such use of these agents may be unfavorable. Oral therapy with the appropriate agents to address this infection in certain patients may be successful. We report a case of graft infection with $E$. meningoseptica causing bacteremia in a dialysis patient who responded to oral agents.
\end{abstract}

Keywords: Bacteremia; Drug resistance; Oral agents

\section{Introduction}

Elizabethkingia meningoseptica is a gram-negative non-fermenting obligate aerobe and is widely distributed in the environment [1]. This organism was formerly called Flavobacterium meningosepticum and the genus Chryseobacterium (was derived from reclassification of members of the genus Flavobacterium) [2]. Although it may present as an opportunistic pathogen in immunocompromised hosts, it is frequently associated with severe infections including meningitis seen in neonates [3] as well as in immunocompetent adults [4, 5]. Other sites of infection associated with this bacteria have included osteomyelitis, pneumonia, endocarditis and skin structure infections [6, 7] mostly occurring in immunocompromised adults. Resistance to many empiric antimicrobial agents commonly used is a classic feature of the organism leading to poor outcome if not addressed in a timely manner. Recognition of this organism is paramount for clinicians to prevent mortality

Manuscript submitted February 27, 2019, accepted March 11, 2019

aDepartment of Infectious Disease, Medical Center of Central Georgia, Macon, GA 31201, USA

${ }^{\mathrm{b}}$ Corresponding Author: Margaret Omatsone, Department of Infectious Disease, Medical Center of Central Georgia, Macon, GA 31201, USA.

Email: margret2402.mo@gmail.com

doi: https://doi.org/10.14740/jmc3274 as a result of lack of effective therapeutic regimens.

Here, we report a case of bacteremia caused by E. meningoseptica in a dialysis patient in whom loss of intravenous access led to rapid transition to oral antibiotic treatment.

\section{Case Report}

A 75-year-old African-American woman with a history of diabetes mellitus, hypertension, heart failure, newly diagnosed end-stage renal disease and antineutrophil cytoplasmic antibody (ANCA)-positive vasculitis was admitted after she has complained of having chills during her hemodialysis and dialysis graft became nonfunctional as a result of which the dialysis was aborted. Her medical history was quite remarkable prior to this time. She was initially admitted 3 months ago for shortness of breath, hemoptysis, and kidney failure. She was later found to have pericardial effusion and subsequently developed respiratory failure. The patient was admitted to intensive care unit (ICU) and ventilated. Kidney disease progressed and biopsy done confirmed p-ANCA associated chronic pauci-immune crescentric glomerulonephritis. She has needed hemodialysis since then and was also treated with cyclophosphamide and steroids. A right arm arteriovenous (AV) graft was placed during that admission and she was subsequently discharged home. At this admission, she complained of some chills and lethargy but no fever or other symptoms. On physical examination, she appeared lethargic. The temperature was $36.4{ }^{\circ} \mathrm{C}$, blood pressure $101 / 51 \mathrm{~mm} \mathrm{Hg}$, pulse 87 beats per minute, respirations 18 breaths per minute and oxygen saturation $97 \%$ on room air. Her heart sounds were regular without any murmurs and lungs disclosed some bibasilar crackles. The right upper arm showed some post-operative changes with mild erythema and was warm to touch around the graft site. Laboratory studies revealed a white blood cell count of 10,110 cells $/ \mathrm{mm}^{3}$, with bandemia of $30 \%$, hemoglobin of $5.7 \mathrm{~g} / \mathrm{dL}$ and platelets of $175,000 / \mathrm{uL}$. The level of sodium was $137 \mathrm{mmol} / \mathrm{L}$, potassium $3.7 \mathrm{mmol} / \mathrm{L}$, bicarbonate $25 \mathrm{mEq} / \mathrm{L}$, urea nitrogen $37 \mathrm{mg} /$ $\mathrm{dL}$ and creatinine $4.8 \mathrm{mg} / \mathrm{dL}$. She was started empirically on cefepime $2 \mathrm{~g}$ intravenously every $24 \mathrm{~h}$. Two sets of blood cultures obtained through a peripheral vein at the time of admission grew gram-negative bacilli after $48 \mathrm{~h}$ of incubation. Blood agar plate showed multiple yellow pigmented colonies which were subsequently identified by the automated bacteriology 
identification system matrix-assisted laser desorption ionization time-of-flight (MALDI-TOF) VITEK mass spectrometry (VMS) as E. meningoseptica. The susceptibility results showed multi-resistance to other agents except ciprofloxacin with minimum inhibitory concentration (MIC) value of $0.5 \mathrm{ug} / \mathrm{L}$, which was added to her regime. A repeat blood culture drawn after 48 $\mathrm{h}$ remained positive. The right arm graft was subsequently removed and gram stain of the graft demonstrated gram-negative bacilli. Final cultures were also positive for E. meningoseptica.

Cefepime was discontinued and only ciprofloxacin was continued for bacteremia. During the course of her hospital stay, she remained afebrile, hemodynamically stable, and no leukocytosis was noted. However, repeat blood cultures after 4 days of therapy still grew the same organism. We decided to add rifampin to her regime. The temporary dialysis catheter placed was also removed and due to loss of intravenous access, medications were given orally-ciprofloxacin and rifampin. The patient's subsequent blood cultures remained sterile after this. She had a new dialysis catheter inserted temporarily and was treated for 4 weeks with oral therapy after discharge. She returned about 1 month later for placement of a new AV graft on the left extremity.

\section{Discussion}

E. meningoseptica is a ubiquitous saprophytic organism found in soil and water. They belong to the class of Flavobacteria which are a rare cause of human disease and are associated with indwelling medical devices [8]. Although it is reported as a cause of meningitis and bacteremia in neonates during the early days of life $[3,8]$, most cases in adults are nosocomial and it frequently affects an immunocompromised host. Important risk factors to note that may predispose to bacteremia by this organism include malignancies, organ transplant, use of immunosuppressants, steroid use, diabetes and renal disease requiring hemodialysis [8-10]. Hsu et al reported in a study carried out in Taiwan from 1999 to 2006, most common underlying diseases were malignancy and diabetes with $60 \%$ of total cases acquiring the infection in ICUs [10]. Our patient described is immunocompromised as she was on therapy for her new diagnosis of vasculitis and also needed hemodialysis putting her at risk for this multi-resistant organism. The association of E. meningoseptica with contaminate medical devices could have attributed to her graft being infected. Her admission to the ICU some weeks ago may have also contributed to how she acquired this organism as it has been isolated in sinks, faucets and drains as well as hospital equipment and environment [11-14]. In addition, outbreaks of this organism have been reported from sources such as saline, lipid, and chlorhexidine gluconate solutions and contaminated sinks $[4,12]$. Arvanitidou [13] reported in a multicenter analysis of dialysis centers that the common isolates from water samples and dialysates in order of frequency were Pseudomonas aeruginosa (22.7\%), E. meningosepticum (14.9\%), Stenotrophomonas maltophilia (13.5\%), Escherichia coli (12.8\%) and Enterobacter cloacae (7.8\%). In this study, gram-negative bacteria were isolated in 141 of the 255 water samples, which further supports the presence of E. meningosepticum in tap (six isolates), treated water (seven isolates), and dialysates (eight isolates). Although the source of infection was not identified for our patient, her exposure to dialysis and risk factors made her susceptible to this organism causing bacteremia and morbidity. The timing from likely exposure in hospital or health facility to acquiring infection with $E$. meningoseptica varies. Some have reported infections that have occurred as early as 5 days from admission to late infections ranging as far as 50 - 70 days after hospital admission $[15,16]$. Our patient possibly acquired her infection during her prior ICU stay which was 42 days before her current presentation.

The resistance to most antimicrobials commonly used for treatment of gram-negative bacteria is one striking feature of this organism. Antibiotic susceptibility profiles have been shown to vary across most reported literature; as a result there is no agreement on appropriate therapy $[4,10]$. It is known to be generally resistant to aminoglycosides and beta-lactam agents including carbapenems $[9,11,13]$. Several mechanisms of resistance have been reported, including the presence of three bla genes which are blaCME coding for the extendedspectrum serine beta-lactamase CME, with blaB and blaGOB genes coding for two unrelated metallo-beta-lactamases (MBLs) which are BlaB (subclass B1) and GOB (subclass B3) respectively $[11,14]$. The MBLs confer resistance to carbapenems while penicillin and cephalosporin resistance could be due to expression of any of the three genes [14].

Susceptible agents to this organism are agents commonly used in treating gram-positive bacteria. In a study reported by Ratnamani and Ratna [16], sensitivity rates of E. meningoseptica isolates revealed $100 \%$ to vancomycin, $100 \%$ to rifampicin, $87.5 \%$ to ciprofloxacin, $75 \%$ to trimethoprim-sulfamethoxazole, $75 \%$ to piperacillin/tazobactam, and $62.5 \%$ to tigecycline, with lower response of $37.5 \%$ to piperacillin, $25 \%$ to tetracycline, and $12.5 \%$ to cefoperazone/sulbactam. Another report showed that vancomycin, rifampicin, newer fluoroquinolones, and minocycline are preferred choices for empiric therapy [11]. Vancomycin, however, should not be considered as the first choice due to high MIC levels and treatment failure reported in literature $[8,16]$.

In our patient, E. meningoseptica was resistant to most agents except for ciprofloxacin and levofloxacin, though vancomycin and rifampicin were not tested in in vitro testing.

The use of combination therapy is not uncommon in treatment of E. meningoseptica and has also been shown to improve clinical response. Most reports involved use of fluoroquinolone to other agents $[8,12,16,17]$.

We initially started our patient on intravenous ciprofloxacin every $12 \mathrm{~h}$ and added rifampicin orally $48 \mathrm{~h}$ later. Ciprofloxacin was also changed to oral therapy on day 3 of her treatment due to loss of intravenous access. Her repeat blood culture $48 \mathrm{~h}$ after commencing combination therapy became sterile. This combination and route was effective in clearing bacteremia with this multi-resistant organism. In similar cases, oral ciprofloxacin with rifampicin should be considered as an option for therapy.

\section{Conclusions}

E. meningoseptica remains a pathogen found in hospital envi- 
ronments leading to infection mostly in immunocompromised hosts. A high index of suspicion is important to note especially in such patients with bacteremia not responding to conventional empiric treatment. Lack of appropriate therapy may result in a fatal outcome. Recognition of this bacterium is important for all clinicians. Further studies in use of oral therapy for treatment may be beneficial.

\section{Acknowledgments}

We thank patient and family for their support with details of information during preparation of this case report.

\section{Financial Disclosure}

Not applicable.

\section{Conflict of Interest}

None.

\section{Informed Consent}

Informed consent was obtained from the patient for publication of this case report.

\section{Author Contributions}

MO is the primary author who wrote the Introduction and case description, and also participated in writing some of Discussion. HK gave guidance on the Discussion of the paper, and is the primary infectious disease consultant managing the patient. $\mathrm{CB}$ helped with writing the Discussion of the paper and searching articles related to the paper. AO searched articles and participated in writing the Discussion and Conclusions of the paper.

\section{References}

1. Henriques IS, Araujo S, Azevedo JS, Alves MS, Chouchani C, Pereira A, Correia A. Prevalence and diversity of carbapenem-resistant bacteria in untreated drinking water in Portugal. Microb Drug Resist. 2012;18(5):531-537.

2. Kim KK, Kim MK, Lim JH, Park HY, Lee ST. Transfer of Chryseobacterium meningosepticum and Chryseobacterium miricola to Elizabethkingia gen. nov. as Elizabethkingia meningoseptica comb. nov. and Elizabethkingia miricola comb. nov. Int J Syst Evol Microbiol. 2005;55(Pt 3):1287-1293.

3. Chiu CH, Waddingdon M, Greenberg D, Schreckenberger PC, Carnahan AM. Atypical Chryseobacterium meningosepticum and meningitis and sepsis in newborns and the immunocompromised, Taiwan. Emerg Infect Dis.
2000;6(5):481-486.

4. Hayek SS, Abd TT, Cribbs SK, Anderson AM, Melendez A, Kobayashi M, Polito C, et al. Rare Elizabethkingia meningosepticum meningitis case in an immunocompetent adult. Emerg Microbes Infect. 2013;2(4):e17.

5. Rao V, Dandamudi R, Koppula P. Elizabethkingiameningoseptica Bacteremia in an Immuno-competent. Patient. JCR. 2017;7:208-210.

6. Weaver KN, Jones RC, Albright R, Thomas Y, Zambrano $\mathrm{CH}$, Costello M, Havel J, et al. Acute emergence of Elizabethkingia meningoseptica infection among mechanically ventilated patients in a long-term acute care facility. Infect Control Hosp Epidemiol. 2010;31(1):54-58.

7. Lee $\mathrm{CH}$, Lin WC, Chia JH, Su LH, Chien CC, Mao AH, Liu JW. Community-acquired osteomyelitis caused by Chryseobacterium meningosepticum: case report and literature review. Diagn Microbiol Infect Dis. 2008;60(1):89-93.

8. Dias M, Fernandes A, Furtado Z. Case series: elizabethkingia meningosepticum. J Clin Diagn Res. 2012;6(9):1550-1551.

9. Shinha T, Ahuja R. Bacteremia due to Elizabethkingia meningoseptica. IDCases. 2015;2(1):13-15.

10. Hsu MS, Liao CH, Huang YT, Liu CY, Yang CJ, Kao KL, Hsueh PR. Clinical features, antimicrobial susceptibilities, and outcomes of Elizabethkingia meningoseptica (Chryseobacterium meningosepticum) bacteremia at a medical center in Taiwan, 1999-2006. Eur J Clin Microbiol Infect Dis. 2011;30(10):1271-1278.

11. Hassan WMNW, Paramasivam R, Kandasamy R, Hassan $\mathrm{MH}$, Zaini RHM. An uncommon Elizabethkingiameningoseptica septicemia in hemorrhagic stroke with septic shock patient during prolonged neuro-intensive care management. Anaesth Pain \& Intensive Care. 2017;21(2):268273.

12. Jean SS, Lee WS, Chen FL, Ou TY, Hsueh PR. Elizabethkingia meningoseptica: an important emerging pathogen causing healthcare-associated infections. J Hosp Infect. 2014;86(4):244-249.

13. Arvanitidou M, Vayona A, Spanakis N, Tsakris A. Occurrence and antimicrobial resistance of Gram-negative bacteria isolated in haemodialysis water and dialysate of renal units: results of a Greek multicentre study. J Appl Microbiol. 2003;95(1):180-185.

14. Gonzalez LJ, Vila AJ. Carbapenem resistance in Elizabethkingia meningoseptica is mediated by metallobeta-lactamase BlaB. Antimicrob Agents Chemother. 2012;56(4):1686-1692.

15. Pereira GH, Garcia Dde O, Abboud CS, Barbosa VL, Silva PS. Nosocomial infections caused by Elizabethkingia meningoseptica: an emergent pathogen. Braz J Infect Dis. 2013;17(5):606-609.

16. Ratnamani MS, Rao R. Elizabethkingia meningoseptica: Emerging nosocomial pathogen in bedside hemodialysis patients. Indian J Crit Care Med. 2013;17(5):304-307.

17. Arbune M, Fotea S, Nechita A, Stefanescu V. Emerging infection with Elizabethkingia meningoseptica in neonate. A case report. J Crit Care Med (Targu Mures). 2018;4(3):96-100. 